\title{
Granulometric composition of electroerosive nichrome powders obtained by electrodispersion of alloy Cr15Ni60
}

\author{
Ekaterina V. Ageeva ${ }^{1 *}$, Alexader Yu. Altuhov ${ }^{1}$, Vladimir I. Serebrovsky ${ }^{2}$ and Valeryi I. \\ Kolmykov $^{1}$ \\ ${ }^{1}$ South-West state University, 305040, Kursk, Russia \\ ${ }^{2}$ Kursk State Agricultural Academy, 305021, Kursk, Russia
}

\begin{abstract}
As a result of electroerosive dispersion of high-chromium steel in lighting kerosene, the results of studying the granulometric composition of powders were obtained. The studies carried out made it possible to establish that the size of the resulting pieces is on average 23.86 microns.
\end{abstract}

\section{Introduction}

The paper presents the results of a study of the microhardness of the W-Ni-Fe 95 sintered electroerosive powder alloy. Powder alloy W-Ni-Fe 95 was obtained by electroerosive dispersion of tungsten-containing waste in a kerosene medium. The resulting electroerosive powder alloy WNF-95 was pressed in a vacuum chamber and sintered by the method of spark plasma sintering. The aim of this work was to study the microhardness of the $\mathrm{W}-\mathrm{Ni}$ Fe 95sintered powder alloy obtained by the electroerosive dispersion of tungsten-containing waste in a kerosene medium [1-5]. It has been established that the microhardness of samples sintered by the method of spark plasma sintering from particles of W-Ni-Fe 95 alloy dispersed by electric erosion obtained in lighting kerosene is $2185.8 \mathrm{MPa}$ and 2268.0 $\mathrm{MPa}$, indicating their suitability for the manufacture of tungsten-nickel-iron alloys [6-10].

The electroerosive dispersion technology, which is characterized by low energy consumption and environmental friendliness, is the most perceptive method for processing most conductive materials, including nichrome waste [1-4].

To develop a technology for obtaining powder nichrome materials by electroerosive dispersion, it is required to carry out comprehensive studies based on both theory and experimental practice to assess the effectiveness [5-9].

It is proposed to use the technology of electrical erosion, currently not used in industry, for the processing of $\mathrm{Cr} 15 \mathrm{Ni} 60$ wastes into powder for the purpose of supplying resources with chromium and nickel. Electrodispersion is environmentally friendly, has no production waste, and small tonnage [10-12].

Electroerosive dispersion (EED) is a technology characterized by low energy consumption and environmental friendliness. The main advantage of the EED technology

\footnotetext{
* Corresponding author: ageeva-ev@yandex.ru
} 
is the possibility of using production wastes, which are cheaper in comparison with pure components. Also, it makes it possible to obtain powder particles from multicomponent alloys [13-17].

The tasks of improving the properties of products can be solved by using new materials. These materials include, first of all, alloy powders. When working with them, it is necessary to study in detail their characteristics, properties, and structure [18, 19].

Powdered products have the disadvantage that the components at different points in the powder are in unequal concentration. This disadvantage can manifest itself, both in the initial state, and persist until the end of production. The preservation of stable and high mechanical properties impose restrictions on the use of these materials in the manufacture of a wide range of parts for various branches of mechanical engineering.. To assess the composition of elements under the influence of electroerosive dispersion, a comprehensive study is proposed [20-22].

The implementation of these studies will solve the problem of obtaining nichrome powders, including nanopowders, and their further use and, thereby, reduce the cost of final production.

The purpose of this work is to study the granulometric composition of electroerosive nichrome powders.

\section{Materials and methods}

Electroerosive nichrome powders were obtained on an electroerosive dispersion unit, protected by RF Patent No. 2449859, from the waste of Cr15Ni60 alloy in distilled water.

During the electrodispersion of the waste of nichrome alloy Cr15Ni60 (Fig. 1), a voltage from a pulse generator was applied to electrodes 2 and 3, consisting of pieces of alloy, and then to the particles of the waste alloy. An electrical breakdown of kerosene 5 in the space between the electrodes occurred when the required voltage level was reached with the formation of the discharge channel 7The material at the discharge point 8 was evaporated and melted together with the lighting kerosene surrounding the discharge channel with the gas bubble 9 under the influence of highly concentrated thermal energy. Due to the dynamic forces in the discharge channel and gaseous decomposition products, the material in the form of drops 4 was ejected outside the discharge zone into the working fluid surrounding the electrodes, and solidified in it, forming spherical particles of the alloy powder.

Investigation of the size distribution of microparticles using a laser diffraction particle size analyzer "Analysette 22 NanoTec".

Dispersion in liquid with ultrasound. Research technique (FR 1.27.2009.06762 "Procedure for measuring particle size in suspensions, emulsions, and aerosols in the nanometer and colloidal ranges using the effect of dynamic light scattering").

Material sample preparation: Samples are dispersed in the process fluid, measuring in the background to reduce the influence of the measuring fluid. To eliminate the influence of the previous measurement, the resulting contamination is measured and eliminated To arrange the particles by size, a sample weighing 1-5 g was placed in a module with a dispersion liquid with a volume of $500 \mathrm{ml}$, automating the measurement when the specified absorption value was reached. 


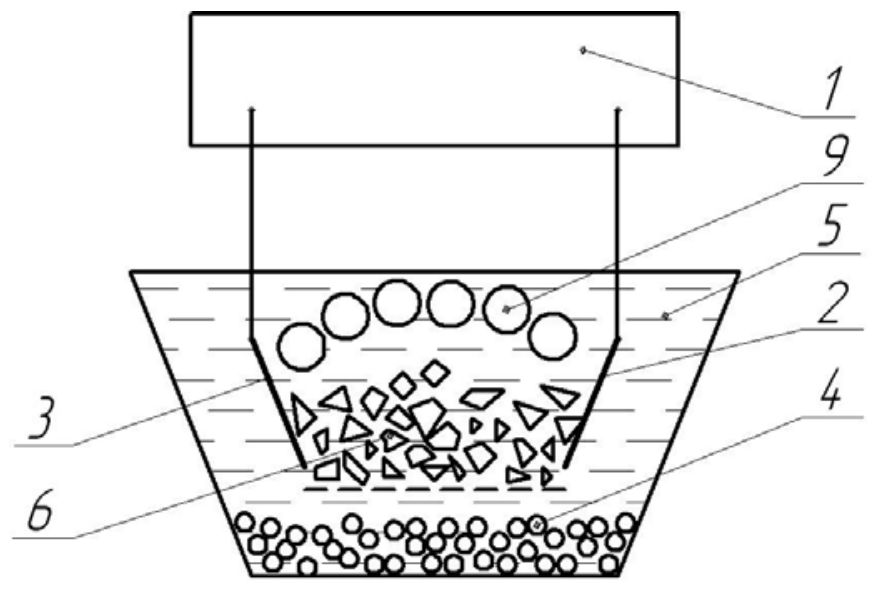

a)

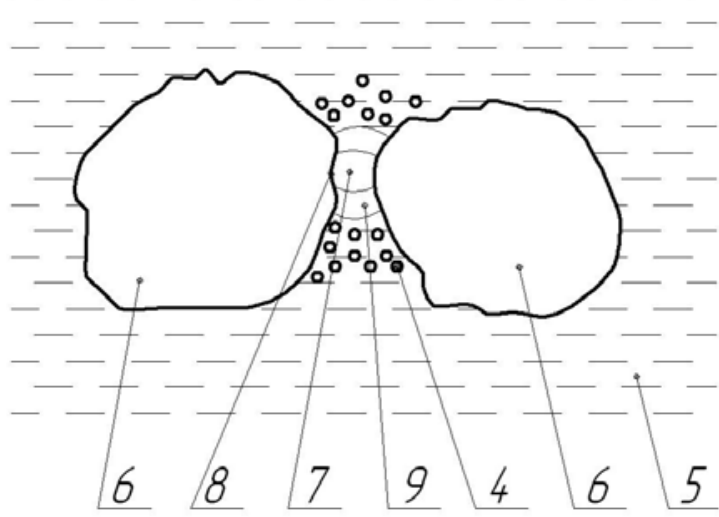

b)

Fig. 1. Electrodispersion process of nichrome alloy Cr15Ni60

a - diagram of the reactor of the installation; b - process diagram

Measurement parameters:

Measurement type - Fraunhofer method; measurement range - $0.1[\mu \mathrm{m}]-1021.87[\mu \mathrm{m}]$; resolution - 102 channels (20/383 mm); absorption - 11.00\%; measurement duration - 90 (scans); regularization is the middle model.

This instrument, which uses laser diffraction to distribute particles in suspensions, emulsions and aerosols, has several advantages over traditional measurement methods. These include a large and accurate measurement range, versatility and reproducibility. The Analysette 22 NanoTec has a measuring range from 0.01 to $2000 \mu \mathrm{m}$. Laser diffraction uses the physical principle of electromagnetic wave scattering in particle size distribution. A laser aimed at the detector through a measuring grating constitutes the structure. The light of the laser beam is scattered by the size of the particles that fall through the dispersing device into the measuring cell, and is focused on the detector by means of a lens.. The volume fractions that correspond to the laser diffraction equivalent diameters are obtained using complex mathematics by calculating the particle size distribution. Difficult to disperse samples due to the built-in ultrasonic bath (volume about $500 \mathrm{ml}$, ultrasound energy and frequency $80 \mathrm{~W} / 36 \mathrm{kHz}$ ) are analyzed without the use of additional equipment. The digital ultrasonic generator always maintains the set power at an optimal and constant 
level. The lower limit of sensitivity for small amounts of small and large particles in their size distributions (within the measurement range) is $3 \%$. Reproducibility according to ISO $13320-1 \mathrm{~d} 50 \leq 1 \%$.

Material sample preparation: Samples are dispersed in the process fluid, measuring in the background to reduce the influence of the measuring fluid. To eliminate the influence of the previous measurement, the resulting contamination is measured and eliminated. To arrange the particles by size, a sample weighing $1-5 \mathrm{~g}$ was placed in a module with a dispersion liquid with a volume of $500 \mathrm{ml}$, automating the measurement when the specified absorption value was reached.

Measurement parameters: Measurement type - Fraunhofer method; measurement range - $0.1[\mu \mathrm{m}]$ - $265.52[\mu \mathrm{m}]$; resolution - 102 channels (20/100 mm); measurement duration 100 (scans); regularization is the middle model.

\section{Results and Discussion}

Figure 2 and Table 1 show the results of a study of the particle size distribution of powders, which were obtained by electroerosive dispersion of a nichrome alloy Cr15Ni60.

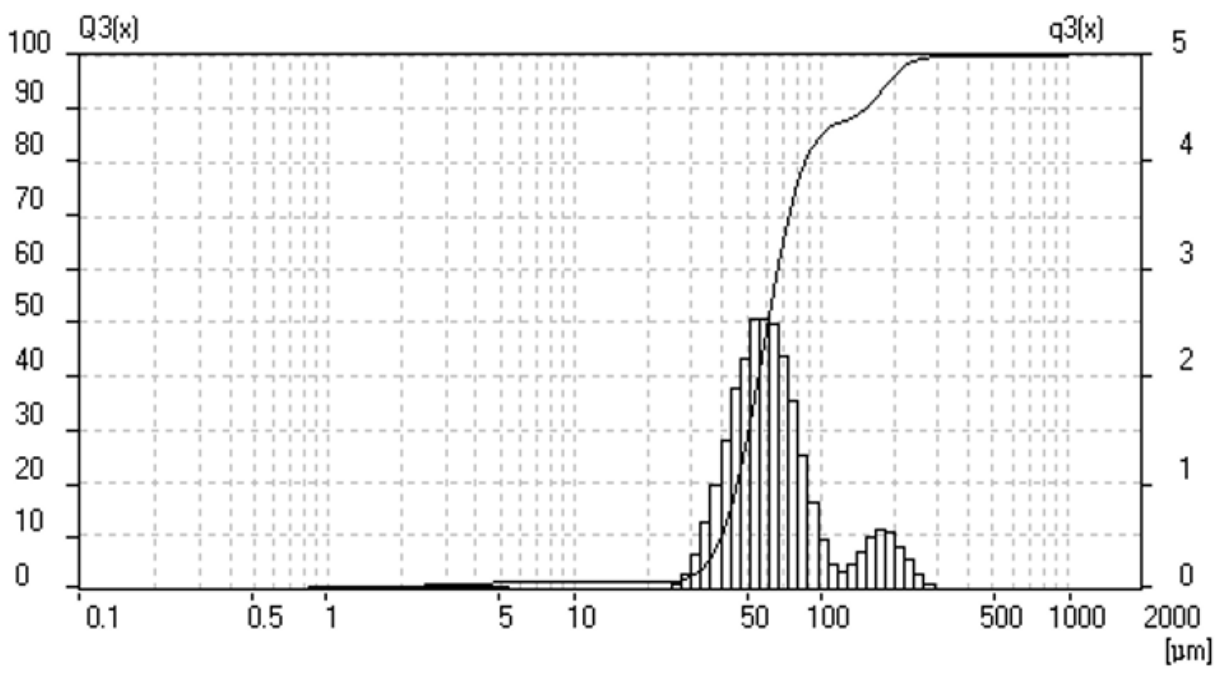

Fig. 2. Powder microparticle size distribution

Integral curve and histogram: integral curve in coordinates Q3 $(\mathrm{x})=\mathrm{f}(\mu \mathrm{m})$ (left scale) each point on the curve, how many\% of the sample has a particle size less than or equal to a given one. Histogram in coordinates q3 $(\mathrm{x})=\mathrm{f}(\mu \mathrm{m})$ (right scale) - amount of sample with a given particle size

Table 1 shows the results of a study of the size distribution of microparticles. 
Table 1. Results of particle size studies

\begin{tabular}{|c|c|}
\hline Parameter & Value $[\mu \mathrm{m}]$ \\
\hline D10 [10\% of particles] & 4.56 \\
\hline D20 [20\% of particles] & 13.51 \\
\hline D30 [30\% of particles] & 16.93 \\
\hline D40 [40\% of particles] & 20.52 \\
\hline D50 [50\% of particles] & 26.99 \\
\hline D60 [60\% of particles] & 34.31 \\
\hline D70 [70\% of particles] & 39.96 \\
\hline D80 [80\% of particles] & 45.41 \\
\hline D90 [90\% of particles] & 52.23 \\
\hline D95 [95\% of particles] & 57.36 \\
\hline $\mathrm{d}[4,3]$ Volumetric average diameter & 23.66 \\
\hline $\mathrm{d}[3,2]$ Average diameter over a surface area & 7.86 \\
\hline $\mathrm{d}[3,0]$ Average diameter in relation to volume & 1.047 \\
\hline $\mathrm{d}[2,0]$ Average diameter in relation to area & 0.64 \\
\hline $\mathrm{d}[1,0]$ Average diameter in relation to length & 0.45 \\
\hline
\end{tabular}

The experiment showed that the average particle size is 23.86 microns.

\section{Conclusion}

As a result of an experimental study aimed at analyzing the granulometric composition of electroerosive powders obtained from waste nichrome alloy $\mathrm{Cr} 15 \mathrm{Ni} 60$, it was revealed that the average particle size is 23.86 microns and $95 \%$ of the total volume of particles in the powder have a size less than or equal to 57.36 microns.

This research work opens up the potential for the application of the method of electroerosive dispersion to obtain a nichrome alloy Cr15Ni60 with a guaranteed particle size distribution, focusing on wastelessness, low tonnage and environmental friendliness.

This work was supported by a scholarship of the President of the Russian Federation (SP940.2021.1).

\section{References}

[1] E.V. Azarova, E.A. Levashov, V.G. Ralchenko, A.P. Bolshakov, E.E. Ashkinazi, Creation of strong adhesive diamond coatings on hard alloy by electric-spark alloying, Metallurgist, 2010, vol. 54, № 7-8, pp. 523-529.

[2] Z. Qiao, X. Ma, W. Zhao, H. Tang, B. Zhao, Nanostructured novel cemented hard alloy obtained by mechanical alloying and hot-pressing sintering and its applications, Journal of Alloys and Compounds. 2008. vol. 462. No. 1-2. pp. 416-420.

[3] S.S. Nayak, S.K. Pabi, M. Wollgarten, J. Banhart, B.S. Murty, Nanocomposites and an extremely hard nanocrystalline intermetallic of al-fe alloys prepared by 
mechanical alloying, Materials Science and Engineering: A, 2010, vol. 527, No. 9, pp. 2370-2378.

[4] K. Maruyama, T. Nonaka, H.Y. Kim, Effects of $\alpha 2$ spacing on creep deformation characteristics of hard oriented pst crystals of tial alloy, Intermetallics, 2005, vol. 13, No. 10, pp. 1116-1121.

[5] A. Shenhar, I. Gotman, S. Radin, P. Ducheyne, Microstructure and fretting behavior of hard tin-based coatings on surgical titanium alloys, Ceramics International, 2000, T. 26, No. 7, pp. 709-713.

[6] A.A. Lipatov, Reactive diffusion in cutting high-alloy steel by means of a hard-alloy tool, Russian Engineering Research, 2013, vol. 33, No. 3, pp. 144-149.

[7] A.M. Adaskin, A.A. Vereshchaka, A.S. Vereshchaka, Study of wear mechanism of hard-alloy tools during machining of refractory alloys, Journal of Friction and Wear, 2013, vol. 34, No. 3, pp. 208-213.

[8] V.L. Bibik, Forecasting of hard-alloyed cutting tool resistance based on thermal diffusivity, Materials Science Forum, 2013, vol. 762, pp. 777-781.

[9] Nawaz W., Xu S., Huang B., Wu X., Wu Z., Li Y., "Nanotechnology and immunoengineering: how nanotechnology can boost car-t therapy“, Acta Biomaterialia. 2020. No. 109. pp. 21-36.

[10] Povarova K.B. Powder Metallurgy of Tungsten Alloys, Proceedings of 3rd EURO PM 2004 Powder Metallurgy World Congress and Exhibition, 2004, No. 5, pp. 106112.

[11] Antsiferova I.V., Esaulova I.A., "Nanotechnology research and education centers as an intellectual basis of nanotechnology in Russia“,Middle East Journal of Scientific Research. 2013. No. 13. pp. 127-131.

[12] Wong K.K.Y., Liu X., "Nanotechnology meets regenerative medicine: a new frontier?“, Nanotechnology Reviews. 2013. T. 2. No. 1. pp. 59-71.

[13] Balykina A.M., Rybalko V.V., Shelamov V.A., "Practical tasks of training of specialists for nanotechnology industry“,International Journal of Nanotechnology. 2019. T. 16. No.6-10. pp. 562-568.

[14] Occhiutto M.L., Costa V.P., Maranhão R.C., Konstas A.G., “Nanotechnology for medical and surgical glaucoma therapy-a review, Advances in Therapy. 2020. T. 37. No.1. pp. 155-199.

[15] Phung C.D., Pham L.M., Jeong J.-H., Yong C.S., Kim J.O., Tran T.H., Nguyen H.T., "Current developments in nanotechnology for improved cancer treatment, focusing on tumor hypoxia“, Journal of Controlled Release. 2020. T. 324. pp. 413-429.

[16] Dar A.H., Rashid N., Dar M.A., Majid I., Hussain S., "Nanotechnology interventions in aquaculture and seafood preservation", Critical Reviews in Food Science and Nutrition“, 2020. T. 60. No. 11. pp. 1912-1921.

[17] Desai P., Thumma N.J., Wagh P.R., Zhan S., Wang J., Prabhu S., Ann D., “Cancer chemoprevention using nanotechnology-based approaches“,Frontiers in Pharmacology. 2020. T. 11. pp. 323.

[18] Choubdar N., Avizheh S., "Nanotechnology based delivery systems of drugs currently used to treat alzheimer’s disease“,Nanoscience and Nanotechnology - Asia. 2020. T. 10. No. 3. pp. 228-247.

[19] Khorev,A.I., "Alloying and heat treatment of structural $(\alpha+\beta)$ titanium alloys of high and superhigh strength“, Russian Engineering Research, 2010, vol. 30, No. 7, pp. 682-688. 
[20] E.V. Ageev, R.A. Latypov, Fabrication and investigation of carbide billets from powders prepared by electroerosive dispersion of tungsten-containing wastes, Russian Journal of Non-Ferrous Metals, 2014, vol. 55, No. 6, pp. 577-580.

[21] E.V. Ageeva, E.V. Ageev, N.M. Horyakova, Morphology of copper powder produced by electrospark dispersion from waste, Russian Engineering Research, 2014, vol. 34, No. 11, pp. 694-696.

[22] E.V. Ageev, R.A. Latypov, E.V. Ageeva, Investigation into the properties of electroerosive powders and hard alloy fabricated from them by isostatic pressing and sintering, Russian Journal of Non-Ferrous Metals, 2015, vol. 56, No. 1, pp. 52-62. 